\section{New diagnosis and subsequent post-partum radiological improvement of lymphangioleiomyomatosis}

A 39-year-old nulliparous woman became breathless after a caesarean section. CT pulmonary angiography (fig 1) showed no filling defect but cysts, small bilateral effusions and thickened interlobular septa were visible consistent with a possible diagnosis of lymphangioleiomyomatosis (LAM). An open lung biopsy performed 11 months later showed that the lung parenchyma contained scattered cysts of various sizes with proliferative smooth muscle bundles in the cyst walls staining for smooth muscle actin and HMB45, which confirmed a diagnosis of LAM.

It is thought that the interstitial infiltrates were secondary to LAM. Cardiac failure may have a similar CT appearance, but the patient had no risk factors apart from recent pregnancy, improved with no anti-failure medication and did not have lung crepitations when auscultated in the immediate postoperative period.

A follow-up CT scan of the thorax 14 months later (fig 2) showed that the reticular shadowing and pleural effusions had disappeared.

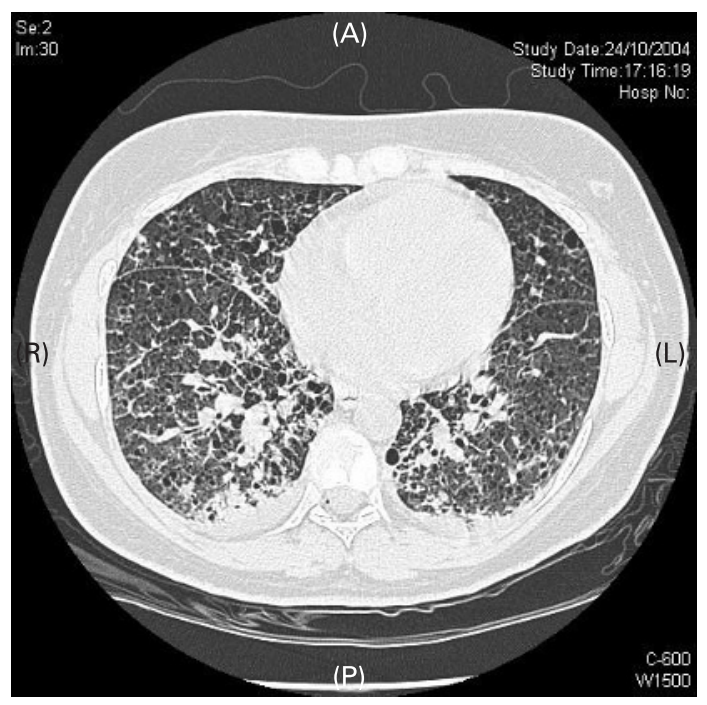

Figure 1 CT pulmonary angiography showing cysts, small bilateral effusions and thickened interlobular septa.

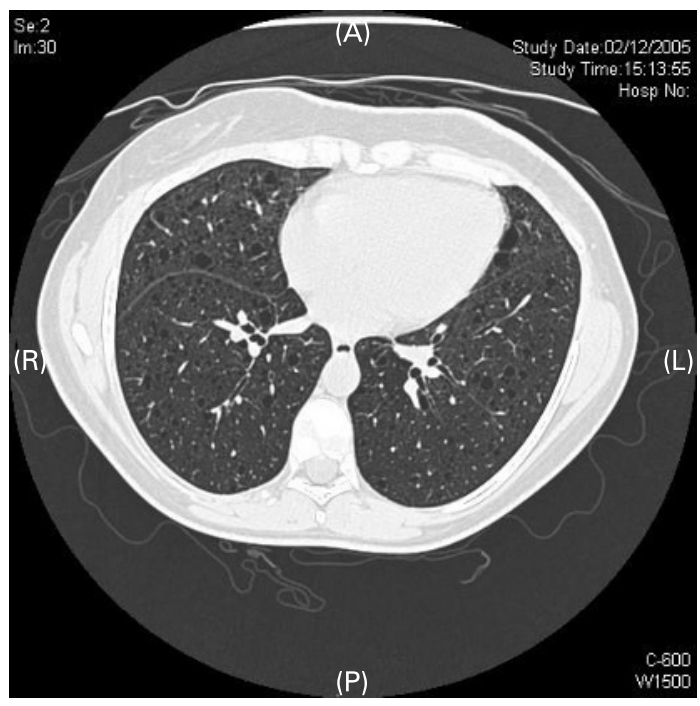

Figure 2 Follow-up CT scan of the thorax.

\section{Learning points}

- Lymphangioleiomyomatosis (LAM) may present for the first time in pregnancy.

- Lymphatic infiltration in LAM may regress post partum.

\section{DISCUSSION}

An 11-fold increase in the incidence of chylous effusions and pneumothoraces in LAM during pregnancy has been described.

\section{G Hagan, P Lawford}

University Hospitals Coventry and Warwickshire, Coventry, UK

Correspondence to: Dr G Hagan, University Hospitals Coventry and Warwickshire, Clifford Bridge Road, Walsgrave, Coventry CV2 2DX, UK; guychagan@hotmail.com

Competing interests: None

Patient consent: Obtained.

Received 25 June 2008

Accepted 3 September 2008

Thorax 2009:64:460. doi:10.1136/thx.2008.103911

\section{REFERENCE}

1. Johnson S, Tattersfield A. Clinical experience of lymphangioleiomyomatosis in the UK. Thorax 2000:55:1052-7. 